\title{
Targeting the vegetarian market with an omega-3 enriched yogurt - but is it acceptable from a sensory perspective?
}

\author{
K. E. Lane, Dr E. J. Derbyshire, Dr W. Li and Dr K. Mahadevan \\ Food, Nutrition and Health Research Centre, Manchester Metropolitan University (MMU), Hollings Campus, Old Hall
}

Lane, Manchester, M14 6HR

Long chain omega-3 polyunsaturated fatty acids (LC3PUFA) have been linked to a spectrum of health benefits ${ }^{(1)}$. Currently, the main sources of LC3PUFA are of marine origin which are unsuitable for vegetarians. In addition, evidence from dietary surveys indicates that LC3PUFA are under-consumed, particularly in vegetarians and vegans ${ }^{(2)}$, indicating a need for innovative solutions. In this pilot sensory study, yogurt was enriched with high LC3PUFA oils, acceptable to vegetarians, as previous research has indicated this type of fortified product may have improved lipid oxidation properties ${ }^{(3)}$.

The aim of this pilot study was to investigate consumer acceptance of fortified yogurt products containing LC3PUFA sources flaxseed and algae oils.

Seven attributes were chosen for natural Greek yogurt including smell, appearance, flavour, texture, consistency, aftertaste and overall acceptance. Samples were prepared using the selected oils in combination with emulsifiers lecithin, Tween 40 and equal amounts of both. Enrichment ratios were calculated in line with previous research. For flaxseed $6 \mathrm{~g}$ of oil was added to $100 \mathrm{~g}$ yogurt and for algae $3.428 \mathrm{~g} /$ $100 \mathrm{~g}$ yogurt. Unfortified Greek yogurt was used as the control. Results were analysed by SPSS ( $v$. 19) ANOVA multiple comparison test (Figures 1 and 2).

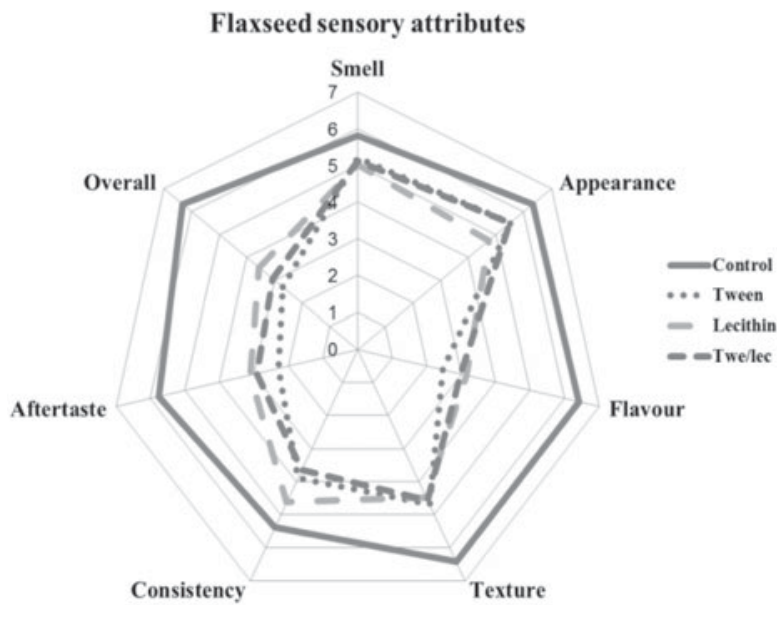

Figure 1: Flaxseed sensory results.

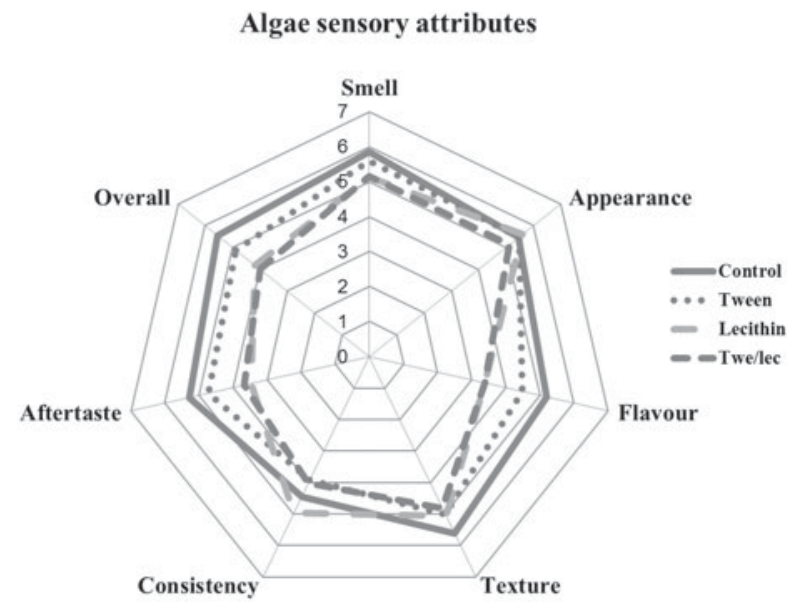

Figure 2: Algae sensory results.

During the flaxseed trial, comments were made in relation to its fishy and bitter taste. Analysis of the sensory results demonstrated that panellists were able to detect flaxseed oil in all the enriched samples for all seven attributes $(P \leq 0.05)$. The algae oil was not as easily detected. The flavour and overall scores for the Tween 40 sample were not significantly different to the control. However, both lecithin (sole and combined) samples received significantly lower scores than the control for all attributes except consistency $(P \leq 0.05)$. Comments made reference to a bitter, sour or acidic aftertaste, but gave no mention of a fishy taste. Sensations of an acidic or bitter aftertaste appeared to occur more frequently in the sole lecithin and combined samples.

Initial sensory analysis trials with a consumer based panel identified that flaxseed oil emulsions can be detected when used to enrich Greek yogurt. The pilot algae oil trial demonstrated that consumers did not detect a significant difference in flavour and overall acceptance when Tween 40 was used as the emulsifier.

1. Ruxton CHS. The benefits of fish consumption. Nutrition Bulletin. 2011; 36(1):6-19.

2. Sanders TA. DHA status of vegetarians. Prostaglandins Leukot Essent Fatty Acids. 2009; 81(2):137-41.

3. Jacobsen C. Enrichment of foods with omega-3 fatty acids: a multidisciplinary challenge. Ann N Y Acad Sci. 2009 Mar; 1190: 141-50. 